\title{
On the Modes of Reaction of Hydrogen Peroxide with some Remarks on the Determination of the Mechanism of a Chemical Reaction from its Kinetics
}

\author{
J. A. CHR IS T I A N S EN
}

Institute of Physical Chemistry, University of Copenhagen, Denmark

It is shown that kinetical experiments prove that the sequence:

$$
\begin{aligned}
& \mathrm{Fe}^{+3}+\mathrm{HO}_{2}^{-} \rightleftharpoons \mathrm{FeOOH}^{+2} \\
& \mathrm{FeOOH}^{+2} \rightleftharpoons \mathrm{Fe}^{+3}+\mathrm{HO}_{2}^{*} \\
& \mathrm{HO}_{2}^{*}+\mathrm{H}_{2} \mathrm{O}_{2} \rightarrow \mathrm{O}_{2}+\mathrm{H}_{2} \mathrm{O}+\mathrm{HO}^{-}
\end{aligned}
$$

where $\mathrm{HO}_{2}^{*}$ is a reactive isomer of $\mathrm{HO}_{2}^{-}$, is the correct expression for the mechanism of the ferric-catalyzed decomposition of hydrogen peroxide

It is further shown that tracer experiments exclude reversible reactions, in which basic ferric ions occur as reactants, as possible partial reactions of the abovementioned catalytic reaction.

Generally it is shown that among a finite number of possible so called kinetic integrals only one, $\varphi(x)$, which is denoted as the chronometric integral can obey the condition $t=\varphi(x)$ in the whole range of $x$ measures the advancement of the reaction.

$\mathrm{H}^{\mathrm{s}}$ aber and Weiss ${ }^{1}$ in their renowned paper introduced the postulate that hydrogen peroxide "is never simultaneously attacked by two monovalent reagents nor by a bivalent reagent whether the material is oxidized to oxygen or reduced to water" 2 .

In other words they deny the possibility that two electrons simultaneously be removed from or taken up by the molecule $\mathrm{H}_{2} \mathrm{O}_{2}$. The statement may also be expressed in the equivalent form that the reactions

and

$$
\begin{aligned}
& \mathrm{O}_{2}^{-2} \rightarrow \mathrm{O}_{2}+2 \mathrm{e} \\
& \mathrm{O}_{2}^{-2}+2 \mathrm{e} \rightarrow 2 \mathrm{O}^{-2}
\end{aligned}
$$


do not occur. The postulate has been repeated by $\mathrm{N}$. Uri ${ }^{2}$ with the addendum that "this conclusion is clearly recognized to-day". This may be true, but the question is whether the recognition is justified. What we are going to discuss below is mainly reactions of the type (A).

There are very old observations, which strongly indicate that reactions which are of the reversed (A) type actually do occur. If e.g. solid silver is treated with oxygen dissolved in an alcaline cyanide solution the initial reaction is

$$
2 \mathrm{Ag}+\mathrm{O}_{2}+4 \mathrm{CN}^{-} \rightarrow 2 \mathrm{Ag}(\mathrm{CN})_{2}^{-}+\mathrm{O}_{2}^{--}
$$

or including the reaction of the strong base $\mathrm{O}_{2}^{--}$with water

$$
2 \mathrm{Ag}+\mathrm{O}_{2}+4 \mathrm{CN}^{-}+2 \mathrm{H}_{2} \mathrm{O} \rightarrow 2 \mathrm{Ag}(\mathrm{CN})_{2}^{-}+\mathrm{H}_{2} \mathrm{O}_{2}+2 \mathrm{HO}^{-}
$$

When the concentration of the peroxide has increased to some extent another reaction sets in, its stoichiometric equation being

$$
2 \mathrm{Ag}^{+}+\mathrm{H}_{2} \mathrm{O}_{2}+4 \mathrm{CN}^{-} \rightarrow 2 \mathrm{Ag}(\mathrm{CN})_{2}^{-}+2 \mathrm{HO}^{-}
$$

Recent experiments by Vibeke Lund ${ }^{3}$ performed in this institute confirmed these results. Mrs. Lund took special case to investigate the stoichiometry at the beginning, when the concentration of peroxide is small. Under these circumstances the stoichiometry was exactly the one represented by the first of the above reaction equations. This can be explained in the simplest way by the assumption of one two-electron step ${ }^{4}$, but the evidence is obviously not sufficient to exclude the two one-electron steps:

$$
\begin{aligned}
& \mathrm{Ag}+\mathrm{O}_{2}+2 \mathrm{CN}^{-} \rightarrow \mathrm{Ag}\left(\mathrm{CN}_{2}^{-}\right)+\mathrm{O}_{2}^{-} \\
& \mathrm{Ag}+\mathrm{O}_{2}^{-}+2 \mathrm{CN}^{-} \rightarrow \mathrm{Ag}(\mathrm{CN})_{2}^{-}+\mathrm{O}_{2}^{-2}
\end{aligned}
$$

or the corresponding reactions including water:

$$
\begin{aligned}
& \mathrm{Ag}+\mathrm{O}_{2}+2 \mathrm{CN}^{-}+\mathrm{H}_{2} \mathrm{O} \rightarrow \mathrm{Ag}(\mathrm{CN})_{2}^{-}+\mathrm{HO}_{2}+\mathrm{HO}^{-} \\
& \mathrm{Ag}+\mathrm{HO}_{2}+2 \mathrm{CN}^{-}+\mathrm{H}_{2} \mathrm{O} \rightarrow \mathrm{Ag}(\mathrm{CN})_{2}^{-}+\mathrm{H}_{2} \mathrm{O}_{2}+\mathrm{HO}^{-}
\end{aligned}
$$

But we may with some confidence conclude from the experiments that the molecular oxygen is reduced to hydrogen-peroxide without breaking of the $\mathrm{O}-\mathrm{O}$ bond.

This conclusion is substantiated by the tracer experiments of Cahill and Taube ${ }^{5}$ and also by $\mathbf{P}$. Baertschi ${ }^{10}$ on reactions of the type (A). These authors conclusively proved that when hydrogen peroxide is transformed by oxydants 
into molecular oxygen, $\mathrm{O}_{2}$ is derived cleanly from the $\mathrm{H}_{2} \mathrm{O}_{2}$. Or in other words by reactions of the two types $(+A)$ and $(-A)$

$$
\mathrm{O}_{2}^{-2} \rightleftharpoons \mathrm{O}_{2}+2 \Theta( \pm \mathrm{A})
$$

there is evidence to show that the $\mathrm{O}-\mathrm{O}$ bond is not broken.

Some years before Haber made his statement kinetic and spectroscopic evidence strongly favoured the assumption of the radicals $\mathrm{HO}_{2}$ and $\mathrm{HO}$ in gas-reactions and there are to-day hardly any experiments to disprove their intermediary existence in certain gas mixtures.

We may therefore with confidence accept them as intermediates in gasreactions and it is quite a natural assumption that they also occur as intermediates in certain reactions especially in aqueous solutions. It is on this idea that the Haber-Weiss mechanism for the catalytic decomposition of hydrogen peroxide and its modifications are based. Curiously enough, at the time when Haber and Weiss started their work the kinetic investigations on the reaction were insufficient and, certainly on account of the tragic events which took place in Habers last days ending with his death in exile, their own experiments were not carried through to the end.

In 1946 I therefore invited V. Sten Andersen to procure the missing kinetic experiments to prove the correctness of the Haber-Weiss mechanism of the decomposition of hydrogen-peroxide with ferric ion as a catalyst. Much to my surprise it turned out however, that neither the Haber-Weiss mechanism in its original form nor a number of different modifications including as intermediates $\mathrm{Fe}^{++}, \mathrm{HO}$ and $\mathrm{HO}_{2}$ would lead to kinetic expressions in conformity with the kinetics found by Sten Ardersen in his very accurate experiments. This forced us to look out for other possible mechanisms and the results are known from the papers by Sten Andersen 6,7 and one by Sten Andersen and myself ${ }^{8}$. In this the following mechanism has been arrived at:

$$
\begin{aligned}
& \mathrm{Fe}^{+3}+\mathrm{HO}_{2}^{-}=\mathrm{FeOOH}^{2+} \\
& \mathrm{FeOOH}^{+2} \rightleftharpoons \mathrm{Fe}^{+3}+\mathrm{HO}_{2}^{-} \\
& \mathrm{HO}_{2}^{*}+\mathrm{H}_{2} \mathrm{O}_{2} \rightarrow \mathrm{O}_{2}+\mathrm{H}_{2} \mathrm{O}+\mathrm{HO}^{-}
\end{aligned}
$$

where $\mathrm{HO}_{2}^{-}$denotes a reactive isomer of the ordinary $\mathrm{HO}_{2}^{-}$-ion.

It must be emphasized that the mechanisms proposed in the papers cited are based directly on the experimental evidence and that the method always has been to follow the reaction till about 95 per cent decomposition. Of course the concentration of hydrogen-peroxide becomes very small at the end, but as well known it can nevertheless be determined with good relative accuracy 
even at small concentrations by the simple device of replacing the ordinary burettes by microburettes for the later determinations, which procedure was adopted.

As can be seen from Sten Andersen's paper 6 the expression log $(a / x)+$ $A(1 / x-1 / a)$ is a linear function of time in the whole interval from $x / a$ $\sim 0$ to $x / a \sim 0.95$, and this is not true for other functions containing a limited number of constants. The deviations at the beginning of the experiment are certainly due to a certain relaxation-time during which the steady state is being established.

Considering that Sten Andersen's results were so unexpected it is no wonder that they have been criticized from different quarters. The criticisms follow two main lines. Some critics maintain that the mechanism for some preconceived reason is untenable. Considering that it turned out to be a necessary consequence of the experiments such objections can only be seriously discussed on the basis of new experimental evidence.

Others have tried to show that Sten Andersen's results can equally well be represented by other functions.

A typical example is found in a review by $\mathrm{N}$. Uri ${ }^{2}$. He maintains that Sten Andersen's results (in ${ }^{6}$ table 1) can be represented by the formula

$$
\log (a-x)-\log (x-x)=k^{\prime} t
$$

where $x=1.77 \cdot 10^{-3} \cdot a$ in ${ }^{6}$ Table 1 is $4.71 \cdot 10^{-2} \mathrm{M}$.

Now in the first place it is evident that this cannot be an exact expression as it would mean that $x$ reaches the limit $x$ at $t \rightarrow \infty$. As however Uri does not pretend the formula to be more than an approximation it is of no use to stress this point.

It is more important that it can be shown that Uri's expression for values of $x$ which are at least a few times greater than $x$ is simply an approximation to Sten Andersen's.

To start with, let us transform to natural logarithms thereby changing $k^{\prime}$ into $k=k^{\prime} / 0.4343$. We may then write the expression

$$
\ln (a / x)+\ln (1-\varkappa / a)-\ln (1-\varkappa / x)=k t
$$

Development of the two last logarithms yields

$$
\ln (a \mid x)+x\left(\frac{1}{x}-\frac{1}{a}\right)+\frac{\varkappa^{2}}{2}\left(\frac{1}{x^{2}}-\frac{1}{a^{2}}\right)+\ldots=k t
$$

Considering that Sten Andersen's last three values of $x$ are respectively 4.02, 2.69 and 1.96 all multiplied by $10^{-3}$ it is thus no wonder that Uri has found that his expression conforms with Sten Andersen's results, when the 
experiments at the lowest peroxide concentrations are omitted. This omission is however not justified by the inaccuracy of the experiments.

It is also questionable whether Uri's statement 2 p. ${ }^{383}$ that the stationary values of ferrous ion and hydroxyl radical are nearly independent of the peroxide concentration, is correct. Unfortunately the mechanism assumed by Uri is not given in his paper and a sound discussion of this point is therefore excluded.

It may however be of interest to the reader to see how a mechanism of the Haber-Weiss type may be treated. Let us assume the following mechanism $(\mathrm{H})$ which is similar to the one used by Barb, Baxendale, George and Hargrave ${ }^{9}$ and which includes the reaction $\mathrm{Fe}^{+2}+\mathrm{H}_{2} \mathrm{O}_{2} \rightarrow \mathrm{FeOH}^{+2}+\mathrm{HO}$ advocated by Uri:

$$
\begin{aligned}
& \mathrm{FeOH}+2+\mathrm{H}_{2} \mathrm{O}_{2} \rightleftharpoons \mathrm{Fe}^{+2}+\mathrm{H}_{2} \mathrm{O}+\mathrm{HO}_{2} \quad\left(\begin{array}{l}
01 \\
10
\end{array}\right) \\
& \mathrm{Fe}^{+2}+\mathrm{H}_{2} \mathrm{O}_{2} \rightleftharpoons \mathrm{FeOH}^{+2}+\mathrm{HO} \quad\left(\begin{array}{l}
12 \\
21
\end{array}\right) \\
& \mathrm{HO}+\mathrm{H}_{2} \mathrm{O}_{2} \rightleftharpoons \mathrm{HO}_{2}+\mathrm{H}_{2} \mathrm{O} \\
& \mathrm{HO}_{2}+\mathrm{FeOH}_{-2}^{+2} \rightarrow \mathrm{O}_{2}+\mathrm{H}_{2} \mathrm{O}+\mathrm{Fe}^{+2}
\end{aligned}
$$

Replacing the symbols $\mathrm{H}_{2} \mathrm{O}_{2}, \mathrm{Fe}^{+2}, \mathrm{HO}, \mathrm{HO}_{2}$ by $\mathrm{X}_{0}, \mathrm{X}_{1}, \mathrm{X}_{2}, \mathrm{X}_{3}$ respectively we may illustrate the situation by the following diagram (Fig. I).

This is intended to mean that the intermediates $X_{1}$ and $X_{3}$ are formed simultaneously from the original stable molecules, $\left[\mathrm{FeOH}^{+2}\right]$ being determined by the acidity of the solution and the total concentration of ferric salt. Furthermore $X_{1}$ disappears by the net reaction (12) and is formed by (31), $X_{2}$ disappears by the net reaction (23) and is formed by (12) and finally $\mathrm{X}_{3}$ disappears by the net reaction (31) and is formed by (23). A stationary flow of intensity $s$ in the direction 123 is obviously equivalent to the occurence of the overall reaction (of speed $s$ )

$$
2 \mathrm{H}_{2} \mathrm{O}_{2} \rightarrow \mathrm{O}_{2}+2 \mathrm{H}_{2} \mathrm{O}
$$

The two flows $o$ indicated must for stoichiometrical reasons be equal. Furthermore the conditions of stationarity are only fulfilled if the net flows $s_{12}, s_{23}$ and $s_{31}$ are all equal and the flow $o$ is zero.

Calling the concentrations of $\mathrm{X}_{1}, \mathrm{X}_{2}, \mathrm{X}_{3}$ respectively $x_{1}, x_{2}, x_{3}$ we thus get four equations

$$
\begin{aligned}
& x_{1} \cdot x_{3}=K_{0}\left[\mathrm{FeOH}^{+2}\right]\left[\mathrm{H}_{2} \mathrm{O}_{2}\right]=P \\
& x_{1} w_{12}-x_{2} w_{21}=s \\
& x_{2} w_{23}-x_{3} w_{32}=s \\
& x_{3} w_{31}=s
\end{aligned}
$$




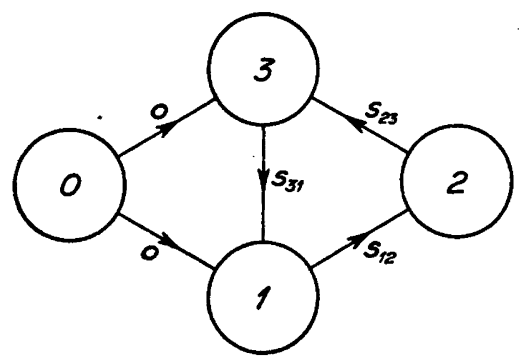

Fig. 1. The diagram shows the flows corresponding to the mechanism (H). For stoichiometric reasons $o_{01}=o_{03}=o$. The steady state conditions furthermore require $s_{12}=s_{23}$ $=s_{31}=s$ and $o=0$.

The symbol $w_{i j}$ is intended to mean the probability pro unit time that the intermediate $X_{i}$ shall react with formation of the intermediate $X_{j}$.

Solution of the last three equations yields

$$
\begin{array}{ll}
\frac{x_{1}}{s}=\frac{1}{w_{12}}+\frac{w_{21}}{w_{12}} \frac{1}{w_{23}}+\frac{w_{21}}{w_{12}} \frac{w_{32}}{w_{23}} \frac{1}{w_{31}} \\
\frac{x_{2}}{s}= \\
\frac{x_{3}}{w_{23}}+\frac{w_{32}}{w_{23}} \frac{1}{w_{31}} \\
\frac{1}{w_{31}}
\end{array}
$$

and by combination with $x_{1} x_{3}=P$ we get

$$
\frac{P}{s^{2}}=\frac{1}{w_{31}} \frac{1}{w_{12}}\left(1+\frac{w_{21}}{w_{23}}+\frac{w_{21}}{w_{23}} \frac{w_{32}}{w_{31}}\right)
$$

Now

$$
\begin{aligned}
& w_{12}=k_{12}\left[\mathrm{H}_{2} \mathrm{O}_{2}\right], w_{21}=k_{21}\left[\mathrm{FeOH}^{+2}\right] \\
& w_{23}=k_{23}\left[\mathrm{H}_{2} \mathrm{O}_{2}\right] ; w_{32}=k_{32} \\
& w_{31}=k_{31}\left[\mathrm{FeOH}^{+2}\right] \\
& P=K_{0}\left[\mathrm{FeOH}^{+2}\right]\left[\mathrm{H}_{2} \mathrm{O}_{2}\right]
\end{aligned}
$$

which by insertion leads to

$$
K_{0} k_{12} k_{31} / s^{2}=\frac{1}{\left[\mathrm{H}_{2} \mathrm{O}_{2}\right]^{2}} \frac{1}{\left[\mathrm{FeOH}^{+2}\right]^{2}}\left[1+\frac{k_{21}}{k_{23}} \frac{\left[\mathrm{FeOH}^{+2}\right]}{\left[\mathrm{H}_{2} \mathrm{O}_{2}\right]}+\frac{k_{21}}{k_{23}} \frac{k_{32}}{k_{31}} \frac{1}{\left[\mathrm{H}_{2} \mathrm{O}_{2}\right]}\right]
$$

The experiments indicate that the last member inside the brackets may be discarded, i.e. that $k_{32} / k_{31}$ is effectively zero.

We may now set $\left[\mathrm{H}_{2} \mathrm{O}_{2}\right]=x$ and $-\frac{\mathrm{d} x}{\mathrm{~d} t}=2 s$. 
Furthermore in a definite experiment $\left[\mathrm{FeOH}^{+2}\right]$ is a constant. We thus arrive at a differential equation of the following form

$$
-k \frac{\mathrm{d} t}{\mathrm{~d} x}=\frac{1}{x} \sqrt{1+\frac{2 x}{x}}
$$

This can be integrated in finite terms, but to compare it with Sten Andersen's expression it is easier to note the fact that so long as $2 x / x$ is small it approximates the form

$$
\begin{gathered}
-k \frac{\mathrm{d} t}{\mathrm{~d} x}=\frac{1}{x}+\frac{x}{\mathrm{x}^{2}} \text { or } \\
k t=\ln (a / x)+\frac{x}{a}\left(\frac{a}{x}-1\right)
\end{gathered}
$$

To estimate the deviations we may use the better approximation

$$
-k \frac{\mathrm{d} t}{\mathrm{~d} x}=\frac{1}{x}+\frac{x}{\mathrm{x}^{2}}-\frac{x^{2}}{2 x^{3}}+\frac{x^{3}}{2 \mathrm{x}^{4}} \ldots \ldots
$$

from which by integration

$$
k t=\ln (a / x)+\frac{x}{a}\left(\frac{a}{x}-1\right)-\frac{x^{2}}{4 a^{2}}\left(\frac{a^{2}}{x^{2}}-1\right)+\frac{x^{3}}{6 a^{3}}\left(\frac{a^{3}}{x^{3}}-1\right)+\ldots
$$

This formula shows that the deviations from Sten Andersen's expression, which comprises only the first two members, become significant when $x / x$ approaches 1. As all Sten Andersen's experiments conform with his expression also when $x / x$ exceeds 1 this indicates that it is his formula and not the one derived above, which is the correct one.

This result has recently been confirmed by the afore-mentioned work by Cahill and Taube ${ }^{5}$. In the above reaction (12) the basic ion $\mathrm{FeOH}^{+2}$ is formed from the peroxide. If now the peroxide oxygen is labelled by ${ }^{18} \mathrm{O}$ the basic ion also becomes labelled. In the back reaction (21) the peroxide is formed from the basic ion which certainly is in isotopic equilibrium with the solvent water. Therefore according to the mechanism (of the Haber-Weiss type) considered above a reversible isotopic exchange between the water and the peroxide must take place, but this would be in contradiction with Cahill and Taubes experiments on the ferric-ion catalyzed decomposition of hydrogen peroxide. To avoid misunderstandings it must be added that the water formed by the overall reaction: $2 \mathrm{H}_{2} \mathrm{O}_{2} \rightarrow 2 \mathrm{H}_{2} \mathrm{O}+\mathrm{O}_{2}$ must be labelled to the same extent as the hydrogen peroxide and the oxygen, but this effect is irreversible.

The tracer experiments show that the oxygen evolved has the same isotopic composition as the one of the original peroxide. The reverse experiment with 
labelled water und unlabelled peroxide to yield unlabelled oxygen has not been performed by $C$. and $T$. so far as can be seen from their paper, but $P$. Baertschi has informed the author, that also in this case the isotopic composition of the oxygen evolved is independent of that of the solvent water ${ }^{11,10}$.

Reverting to kinetics it can be proved that any mechanism leading to an expression, which is different from Sten Andersen's must be discarded. As the proof may be of interest also in other cases we shall try to present it in a general form.

We restrict our considerations to cases where one number $(x)$ is sufficient to describe the advancement of the reaction.

Let us denote a function which has been derived by integration of the expression for the reciprocal steady state velocity derived from some reaction mechanism as a kinetic integral (belonging to the mechanism in question).

Any kinetic integral must obviously have one of the following two equivalent properties:

1) It approaches zero when $x$ approaches a constant $(a)$ and it approaches infinity, when $x$ approaches zero or 2) It approaches zero when $x$ approaches zero and it approaches infinity when $x$ approaches a constant $(a)$.

(In Sten Andersen's experiments $x$ has been so defined that it is the condition in the form 1) which must be fulfilled).

If now a kinetic integral $\varphi(x)$ has been shown by experiments to obey the condition

$$
t=\varphi(x)(a>x>0)
$$

where $t$ is the time, we shall denote it as the chronometric integral (of the overall reaction in question).

Thus several but a finite number of different kinetic integrals may belong to one overall reaction. What we intend to prove is that only one of these can be the chronometric integral of that reaction.

The following statement must necessarily be true: Two different kinetic integrals containing the same limited number of adjustable constants may be made to coincide in a limited range of $x$ by a suitable choice of the constants, but it is impossible to make them coincide in the complete range from $a$ to zero.

If therefore a kinetic integral $\varphi$, containing only few adjustable constants has been proved by experiments to obey the condition $t=\varphi$ in the whole range of $x, \varphi$ must be the chronometric integral of the reaction and no other satisfactory one can be found.

As now nearly any change in the mechanism, from which the chronometric integral has been derived, leads to a definite change in the form of the integral, 
the mechanism from which it has been derived must be in nearly all cases the only one which is in agreement with the experiments.

It must be remembered that not all values of the constants are acceptable. In the first place the constants $k_{i j}$ can never be negative. Furthermore, especially when their dependence on temperature is also investigated, it may turn out that they have impossibly large or small values. In such cases modifications of the mechanism must be looked for.

To quote an example: By extending his investigation of the catalytic decomposition of hydrogen-peroxide to different temperatures Sten Andersen 7 found for the mechanism $(\mathrm{C})$ :

$$
\begin{aligned}
& \mathrm{Fe}^{+3}+\mathrm{HO}_{2}^{-} \rightleftharpoons \mathrm{FeOOH}^{+2} \\
& \mathrm{FeOOH}^{+2} \rightleftharpoons \mathrm{FeOH}^{+2}+\mathrm{O} \\
& \mathrm{O}+\mathrm{HO}_{2}^{-} \rightarrow \mathrm{O}_{2}+\mathrm{HO}^{-}
\end{aligned}
$$

that the ratio $k_{50} / k_{52}$ was of the order of magnitude $10^{9}$ and was nearly independent of temperature. Now from our general knowledge of the numerical values of velocity-constants this result is extremely improbable. This compelled us to introduce the modified mechanism (D) (compare p. 1058):

$$
\begin{aligned}
& \mathrm{Fe}^{+3}+\mathrm{HO}_{2}^{-} \rightleftharpoons \mathrm{FeOOH}+2 \\
& \mathrm{FeOOH}^{+2} \rightleftharpoons \mathrm{Fe}^{+3}+\stackrel{*}{\mathrm{HO}_{2}^{-}} \\
& \stackrel{*}{\mathrm{HO}_{2}^{-}}+\mathrm{H}_{2} \mathrm{O}_{2} \rightarrow \mathrm{O}_{2}+\mathrm{H}_{2} \mathrm{O}+\mathrm{HO}^{-}
\end{aligned}
$$

The assumption of mechanism (D) leads to a kinetic integral of exactly the same form as the one derived from mechanism (C) but now the ratio $k_{50} / k_{52}$ becomes of the order of magnitude 1 and is still nearly independent of temperature, which is what we should expect from general knowledge.

To-day it may be added that the experiments by Cahill and Taube ${ }^{5}$ definitely exclude the possibility (C) as the reaction

$$
\mathrm{FeOOH}+2=\mathrm{FeOH}^{+2}+\mathrm{O}
$$

and its reverse would cause a reversible isotopic exchange between hydrogen peroxide and the solvent water.

All the experiments thus go together to show that the mechanism (D) represents the way in which the ferric-catalyzed decomposition of hydrogen peroxide takes place. 


\section{REFERENCES}

1. Haber, F., and Weiss J. Proc.Roy.Soc. (London) A 147 (1934) 332.

2. Uri, N. Chem.Revs. 50 (1952) 375.

3. Lund, V. Acta Chem. Scand. 5 (1951) 555.

4. Raschig, F. Metall u. Erz. 25 (1928) 467, 525.

5. Cahill, A. E., and Taube, H. J. Am. Chem. Soc. 74 (1952) 2312.

6. Andersen, V. S. Acta Chem. Scand. 2 (1948) 1.

7. Andersen, V. S. Acta Chem. Scand. 4 (1950) 914.

8. Christiansen, J. A., and Andersen, V. S. Acta Chem. Scand. 4 (1951) 1538.

9. Barb, W. G., Baxendale, J. H., George, P., and Hargrave, K. R. Trans. Faraday Soc. 47 (1951) 591.

10. Baertschi, P. Experientia 7 (1951) 215.

11. Baertschi, P. Unpublished experiments. 